\title{
Intelligent geographic information platform for transport process analysis
}

\author{
O. Golovnin ${ }^{1}$, A. Fedoseev ${ }^{1,2}$, T. Mikheeva ${ }^{1}$ \\ ${ }^{1}$ Samara National Research University, 34 Moskovskoe Shosse, 443086, Samara, Russia \\ ${ }^{2}$ Space-Rocket Centre Progress, 18, Zemetsa str., 443009, Samara, Russia
}

\begin{abstract}
We developed an intelligent geoinformation platform for transport process analysis. The paper describes a purpose and functions of the synthesized platform as well as its structure including components and tools. We used the intelligent transport geoinformation platform for solving problems of acquisition, storage, processing and analysis of objects, processes and phenomena of urban transport infrastructure. The paper presents results of simulation and full-scale experiments.
\end{abstract}

Keywords: intelligent transport system; geoinformation system; traffic flow; transport infrastructure, ITSGIS; GIS; ITS

\section{Introduction}

Improvement of economy, comfort and traffic safety is promoted by the use of modern systems for transport processes analysis, including [1,2]: information systems of Vehicle-to-Infrastructure class, automated traffic control systems and intelligent transport systems. These systems are based on modern achievements and innovations in the field of transport management $[3,4]$ : information support for traffic participants, transport detectors and radars, meteorological information systems and video surveillance systems, traffic lights, which include intelligent neural networks [5] and genetic [6] algorithms, intelligent pedestrian crossings and information displays on road forks.

In terms of complex intelligent transport systems development, information sources determine the heterogeneity of transport processes data. These information sources also cause heterogeneity of both hardware and software platforms, which leads to significant reduction in the efficiency and relevance of the data and decision-making procedures [7, 8]. All the factors mentioned above ensure consistency and relevance of developing special means for transport process analysis that makes it possible to compensate the heterogeneity of information spaces.

High efficiency in the study of transport processes can't be achieved without the spatial reference of numerous static and dynamic objects that compose the transport infrastructure $[9,10]$. Storage and manipulation of geospatial and attribute data that describe the objects, processes and phenomena of the transport infrastructure can be implemented with a high degree of efficiency in the environment of the geoinformation system [11,12]. Geoinformation system allows constructing a geoinformation model of the transport network in an urbanized area reflecting all changes in the real world transport infrastructure [14]:

- changes in the infrastructure component of the street-road network: overlapping lanes, narrowing the roadway when performing road works, reconstruction, construction of new residential areas, shopping and entertainment centers and other points of attraction;

- changes in the deployment of traffic management facilities: implementation of temporary schemes of traffic management, modernization of traffic lights, installation of new traffic lights and road signs, introduction of dedicated lanes for public transport.

Thus, the goal of this work is to develop intelligent algorithms and software that form an integrating platform based on the geoinformation system that allows solving heterogeneous tasks of transport process analysis in a single operating environment:

- monitoring the characteristics of traffic flows, street-road network, traffic management facilities, environment;

- organization of freight and passenger transportation: analysis of transport demand, construction of traffic routes, reduction of resources spent, increase in profits;

- optimal management (local and global): development of the transport network of regions and megacities, interaction of various modes of transport, reducing transport delays, necessary and sufficient deployment of traffic management facilities;

- safety improvement: pre-crash restraint, risk of accidents reduction, accident incidence rate decrease.

\section{Purpose, capabilities and structure of the platform}

Functional capabilities of the transport analysis platform cover the whole range of tasks posed in the management of transport processes $[15,16]$ :

- monitoring of objects, processes and phenomena of transport infrastructure;

- organization of freight and passenger transportation;

- optimal management of transport infrastructure and traffic flows;

- safety.

The platform is built on a modular principle: each task or part of it is implemented as a subsystem (module). All subsystems are implemented on the basis of a single tool environment - the core of the system. The core is a universal software infrastructure that includes a set of software components, modules and a georeferencing database. 
Image Processing, Geoinformation Technology and Information Security / G O. Golovnin, A. Fedoseev, T. Mikheeva

Functional specification of the core includes:

- formation, processing and storage of a database;

- multithreaded processing of data by algorithms;

- $\quad$ ensuring interaction with the geographic information system;

- $\quad$ provision of interaction with subsystems and between them, other systems;

- $\quad$ provision of platform integration capabilities in various public and network services (internet portals);

- import of static data from other formats and systems;

- export data to external formats

- $\quad$ providing information to the operator in a graphic form for decision making [17].

Functional specification of the monitoring subsystem includes:

- obtaining information from assessment, measuring, meteorological information systems and processing of survey data;

- transfer of operational attribute information to the georeferencing database;

- definition of parameters of fundamental diagrams for sections of the road network, characteristics and composition of traffic flows;

- calculation of the forecast of the development of the situation [18];

- response to the results of self-diagnosis of technical monitoring and management tools;

- formation and transmission of events to the control subsystem.

Functional specification of the control subsystem includes:

- reception and processing of events coming from the monitoring subsystem;

- the definition of the management and information scenario;

- transformation of the control actions generated by the control subsystem into the format of actuators (traffic light controllers, variable information signs);

- transmission of control signals to actuators.

Functional specification of the subsystem of information support of the traffic participants includes:

- conversion of the control actions developed by the control subsystem to the format of the information support facilities for the traffic participants (web service, SMS-distribution);

- $\quad$ providing access to public elements of visualization and information services platform through the website;

- ensuring the functioning of the Internet services of the platform on mobile devices.

The territorial remoteness of platform users, the interest in different functional components, the use of their own data warehouses determine the distributed architecture of the platform [19, 20], in which the interaction is carried out by means of local computer networks or the Internet using a central server (Fig. 1). Access of interested persons (users) to data is limited by their spheres of influence [21].

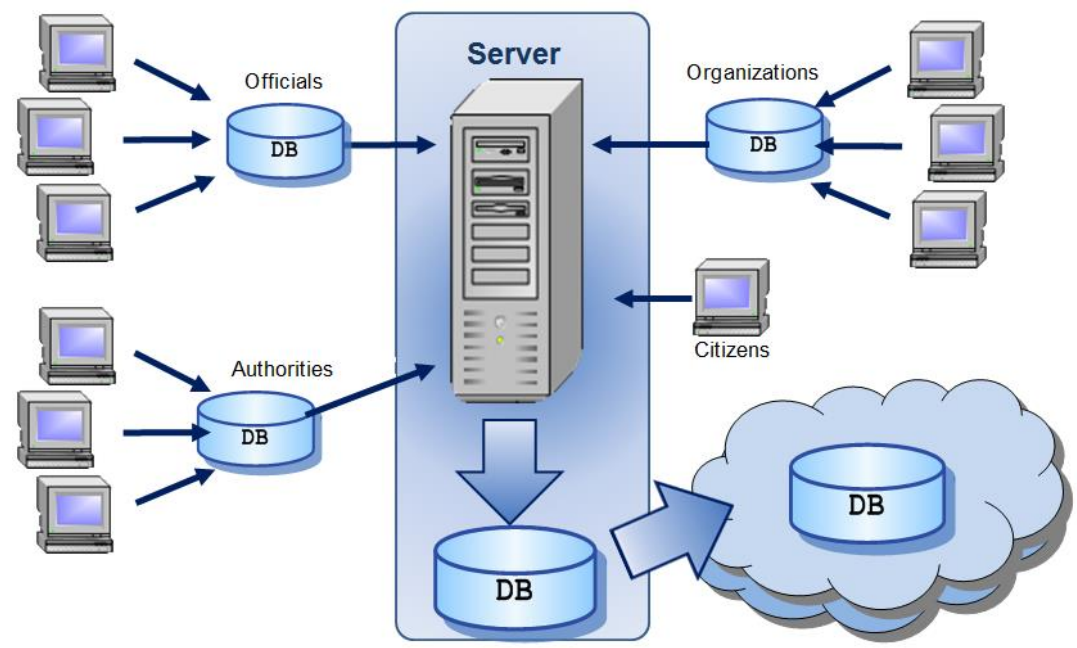

Fig. 1. Scheme of interaction between platform users.

The platform includes:

- database server with a database management system supporting geospatial objects;

- $\quad$ application server;

- client applications.

The database management system provides secure storage and manipulation of data, maintaining their integrity, replicating. The server is a host for hosting platform services and their business logic, provides multi-user work, authorization and delineation of access rights for clients and subsystems, compression and encryption of data, maintaining connections with customers. Client applications provide end-user access to platform services. Subsystems that perform a task are embedded in the database, application server, and client applications using the developed methods for securely connecting / disconnecting subsystems.

The georeferencing database is built on the basis of the "Virtual Database" pattern and is an integrated repository of attribute and geospatial data, including: 
- $\quad$ electronic basis of the map of the urbanized territory;

- directories and registers;

- geoobjects of transport infrastructure, implemented by connected subsystems.

The virtual database of the platform supports topological relationships in queries, ensures execution of both SQL and LINQ. The requirement of interaction with third-party subsystems and data sources dictates the need to apply open standards in the database used for storage, processing and transmission of geospatial information. As such standards, the platform uses OGC specifications and standards [22].

The PostgreSQL database management system with the PostGIS geospatial extension used to store geospatial and attribute information. The extension defines a special type of spatial data - a full set of functions and indexes for working with them. The introduction of additional relational tables into the database is provided by the version subsystem of database structure migration: when adding tables, a transaction is created that makes changes to the existing data schema and updates the version of the schema; when you remove the tables, the reverse transaction is executed.

One of the functional components of the platform is the ITSGIS geoinformation system. ITSGIS provides a platform for analyzing transport process with tools for viewing an electronic map with applied objects with the ability to connect / disconnect map layers, scale, and select geodata.

The reference of transport infrastructure objects to an electronic map is determined by the "Geometry" entity that migrated from the ITSGIS core as an OGC geometry in the Well-known Binary format. Geometry is equipped with a description of the visualization style and has a reference

to the layer of the electronic map (for example, for the "Road Sign" object, this layer is the "Posts" layer.

The transport infrastructure objects reference is defined by the "Geometry" entity, migrated from ITSGIS' core and represented by OGC-geometry in the format of "Well-known Binary". Geometries possess visualization style descriptions and are bound to an electronic map layer (for example, a "Road sign" object is bound to the "Posts" layer).

Objects can possess a description of geometry's address ing, describing such address components as "Country", "Region", "City", "District", "Street”, "Highway", "Landmark". A "Landmark" is commonly represented by a house number, a picketage or a verbal description.

Requirements for security, reliability and interoperability of the application server platform lead to the need to build a crossplatform unified transaction model. In the developed platform, this model provides a set of technologies Windows Communication Foundation. The application server delineates the access rights of users of the system based on the geo-role subsystem: the right to view / modify information is determined both by taking into account the layer of the electronic map and the polygonal area on the map. Additional services and their business logic are connected to the application server during the configuration of the server using the IoC container. The container registers all additional services, models, data access layers, domain and auxiliary objects. When the application server is started, dependencies between objects are automatically resolved, additional services are created and launched.

Client applications are implemented as "thick" (Microsoft .NET application with WinForms and WPF interfaces for the CLR runtime) and "thin" (web application on the Yii2 platform with an interface implemented in the browser environment using HTML, CSS and JavaScript) clients. All client applications provide the connection of additional modules (subsystems) and flexible configuration of the user interface. The client consists of freely connected modules that are dynamically detected and compiled into a single unit at runtime. Modules contain both visual and non-visual components, representing different vertical layers of the platform.

\section{Toolware of the platform}

Based on the tasks to be solved, the software modules of the platform for transport process analysis, built using the "Embedded extension" pattern, are implemented for the C \# and XAML runtime CLR.

Modules (subsystems) are aimed to solve monitoring tasks:

- Plugin Yamgis - module "Monitoring";

- PluginIntensity - module "Characteristics of traffic flows".

Modules (subsystems) are aimed to solve the problems of freight and passenger transportation:

- PluginUds - the module "Street-road network";

- PluginRoute - the module "Routes".

Modules (subsystems) are aimed to solve management problems:

- PluginPassport - module "Management of transport infrastructure";

- PluginSimulation - module "Modeling".

Modules (subsystems) are aimed at solving security tasks:

- PluginDTP - module "Road accidents";

- PluginFirecenter - module "Accident clusters".

The following modules (subsystems) have been developed for storage, processing and analysis of data on transport infrastructure objects:

- PluginInfo - module "Organizations on the map";

- PluginPetrolstation - module "Gas station";

- PluginRWC - module "Railway crossings";

- PluginPost - module "Road signs and traffic lights"; 
Image Processing, Geoinformation Technology and Information Security / G O. Golovnin, A. Fedoseev, T. Mikheeva

- PluginRoadmarking - module "Road marking";

- PluginBarricado - module "Fences";

- PluginBusStop - module "Public transport stops";

- PluginCablenetwork - module "Artificial lighting".

To obtain primary cartographic information about the transport infrastructure, the ITSGIS geoinformation system [23] and the cartographic service Open Street Map [24] are used. Data on the transport infrastructure in ITSGIS are presented in the form of detailed layers of the electronic map with exact geometric parameters of the street-road network and are used to form the model of the graph of the street-road network. Geoobjects are stored and processed in a format and according to requirements that conform to the OGC specifications [22]. Each class of objects that have a spatial reference has its own layer of the electronic map. The Open Street Map data is stored as OSM format files with a description of the road segments [24] that are used to compile the cartographic base in ITSGIS. ITSGIS includes the program module ITS.MapConverter, which converts data of various formats (including OSM) to the form used by ITSGIS, and vice versa.

The web application for the provision of traffic information for the Yii2 platform with an interface implemented in a browser environment using HTML, CSS and JavaScript was developed. ITSGIS provides access to the geodata for the Web application in the form of XML documents and tiles, which are displayed by the OpenLayers 2 module.

Features of the physical implementation of the system are described by the component diagram shown in Fig. 2.

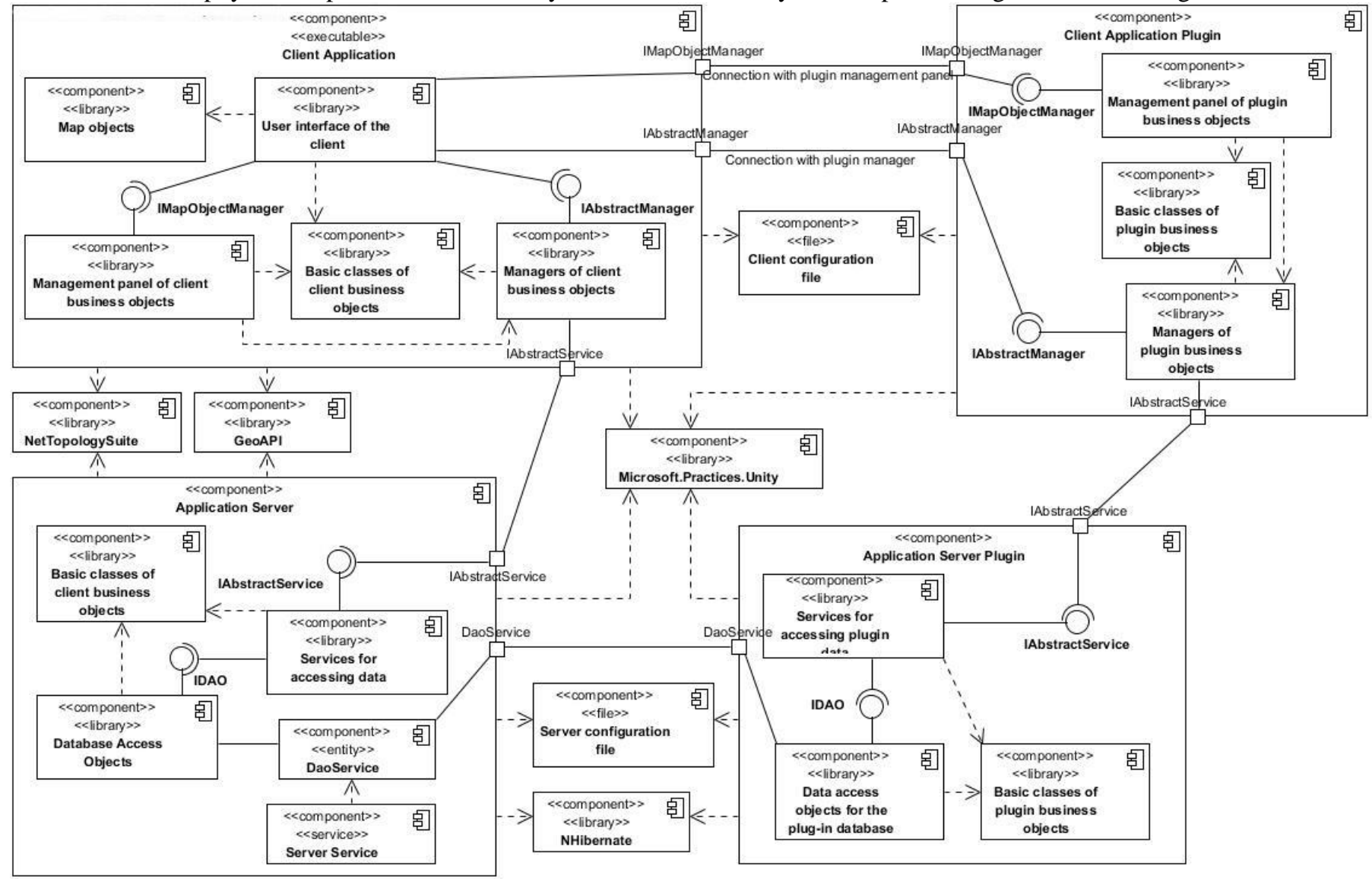

Fig. 2. Component diagram of the platform.

To represent the topology of the developed transport analysis platform, a deployment diagram was constructed (generally, Fig. 3). At the heart of the system is a multi-level architecture in which levels are distinguished: data processing (database server), business logic (application server based on the WCF technology) and presentation to the user (clients).

Modified deployment method provides access to the system through the site-geoportal from the web browser. In this case, the system nodes are:

- "app.itsgis server" - the server on which the application server and the geoserver are deployed;

- "web-portal server" - the geoportal application server on which Apache and MySQL components are deployed;

- "client" - the end-user computer on which the web browser is installed.

\section{Deployment of the platform for solving problems of transport process analysis}

\subsection{Transport management tasks}

The task of studying local control in conjunction with a point zone (Fig. 4) is associated with the method of local control of transport flows at a separate intersection. 


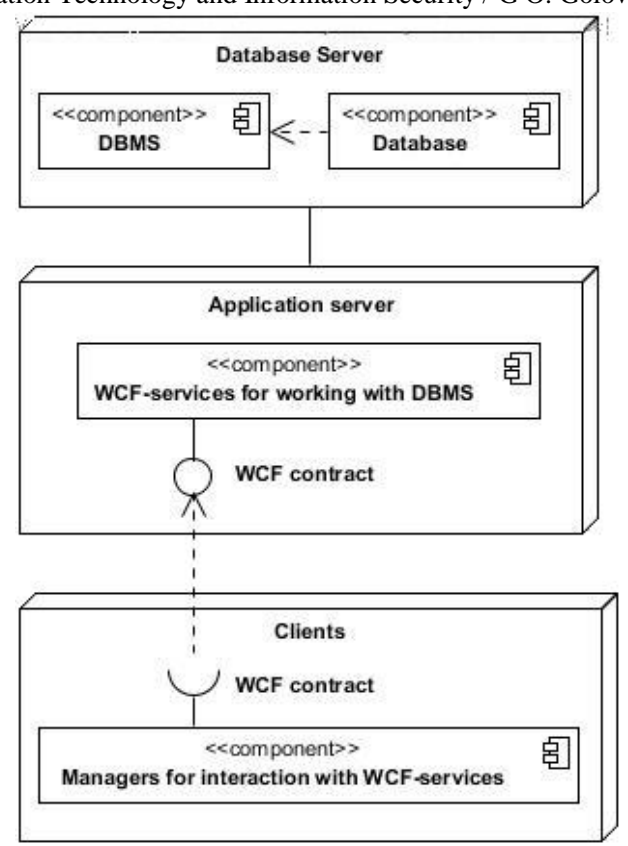

Fig. 3. Deployment diagram of the platform.

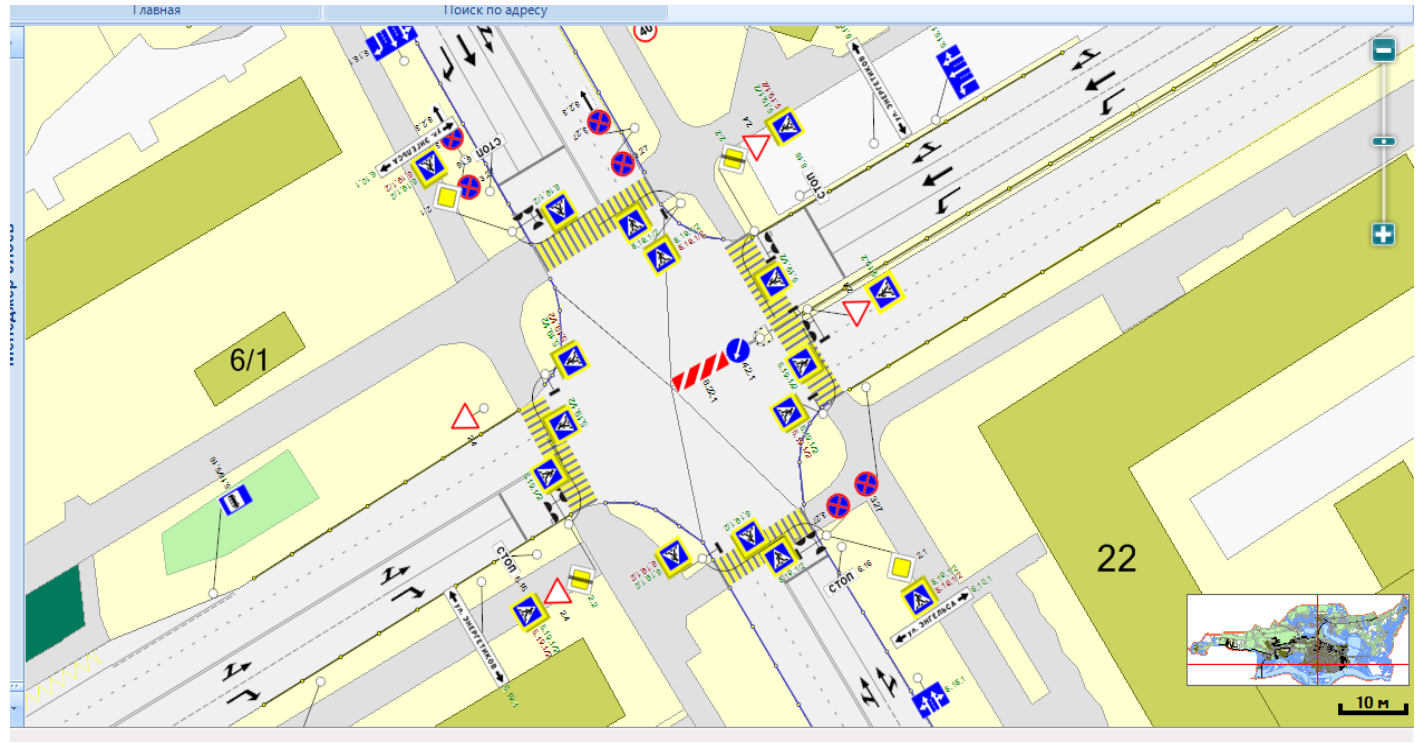

Fig. 4. Functional zone of local control.

Co-ordinated control methods involve the optimization of transport processes on the highway, the control zone will be defined in this case by linear decomposition.

System zonal management affects several classes of transport infrastructure objects, united by the task being solved, and therefore spatial zoning will be defined by the polygon (Fig. 5).

A lot of sections of the street-road network contain subsets of distances, intersections, pedestrian crossings, railway crossings, overpasses and tunnels for solving the problems of studying traffic flows in these sections when describing them by a planar graph. Fig. 6 shows a multi-level transport interchange.

\subsection{Traffic flow intensity research}

The layer of the electronic map showing the intensity of traffic flows contains the mean annual daily intensity at intersections (Fig. 7).

The graph of the street-road network of the local intersection is characterized by the intensity of traffic flows (Figure 8). The color indicates the power intensity on this arc of the graph of the street-road network. The platform uses the "Divergent color scheme" pattern to visualize the intensity of traffic flows. For the central value of the intensity of the traffic flow, the capacity of the section of the road network is assumed. The intensity values above the center value are displayed in shades of red, values below the central level are displayed in shades of green. The use of a non-standard color scheme for geoinformation systems is conditioned by the specifics of the subject area and the following analogies: the green signal of the traffic light allows movement - low intensity will allow the road network to pass freely, the red signal of the traffic light prohibits traffic - a high intensity value will not allow the passage without forced stops. 


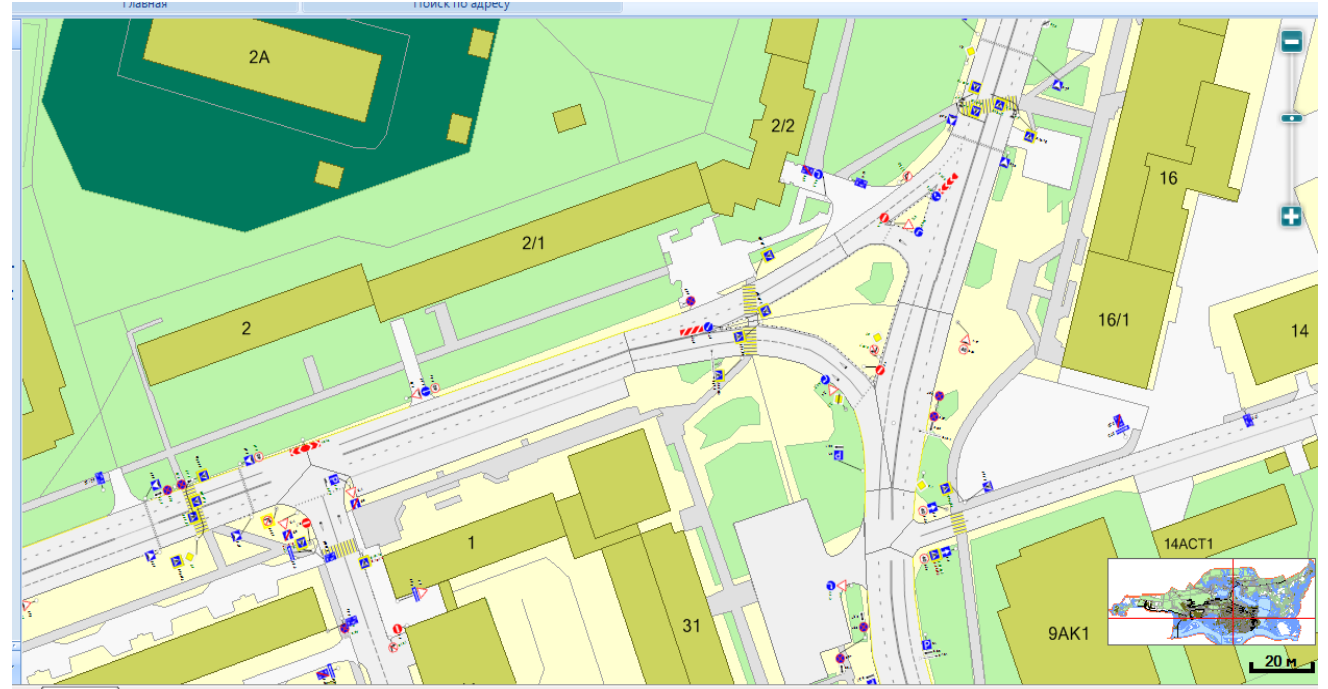

Fig. 5. Functional zone of system control

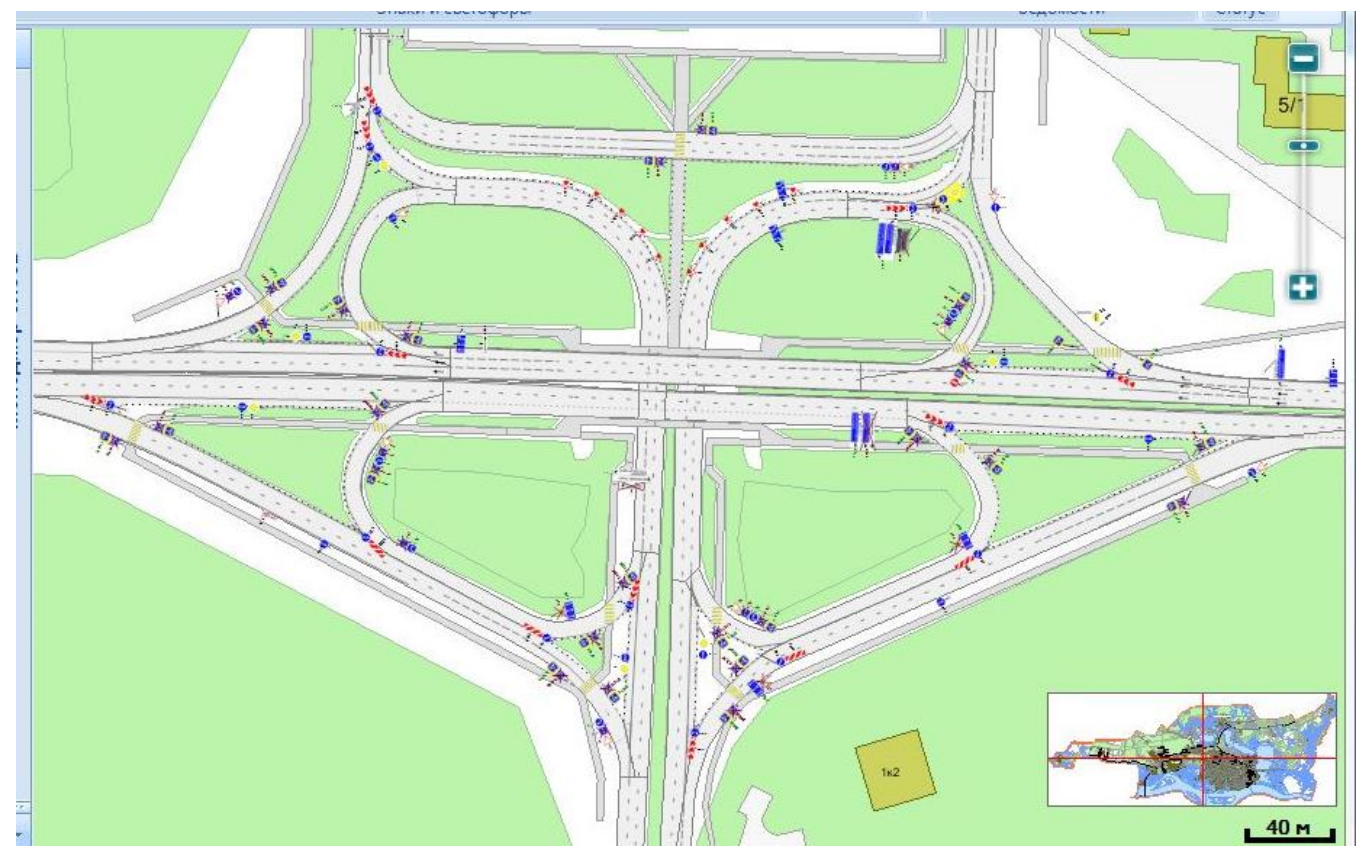

Fig. 6. Multi-level road junction.

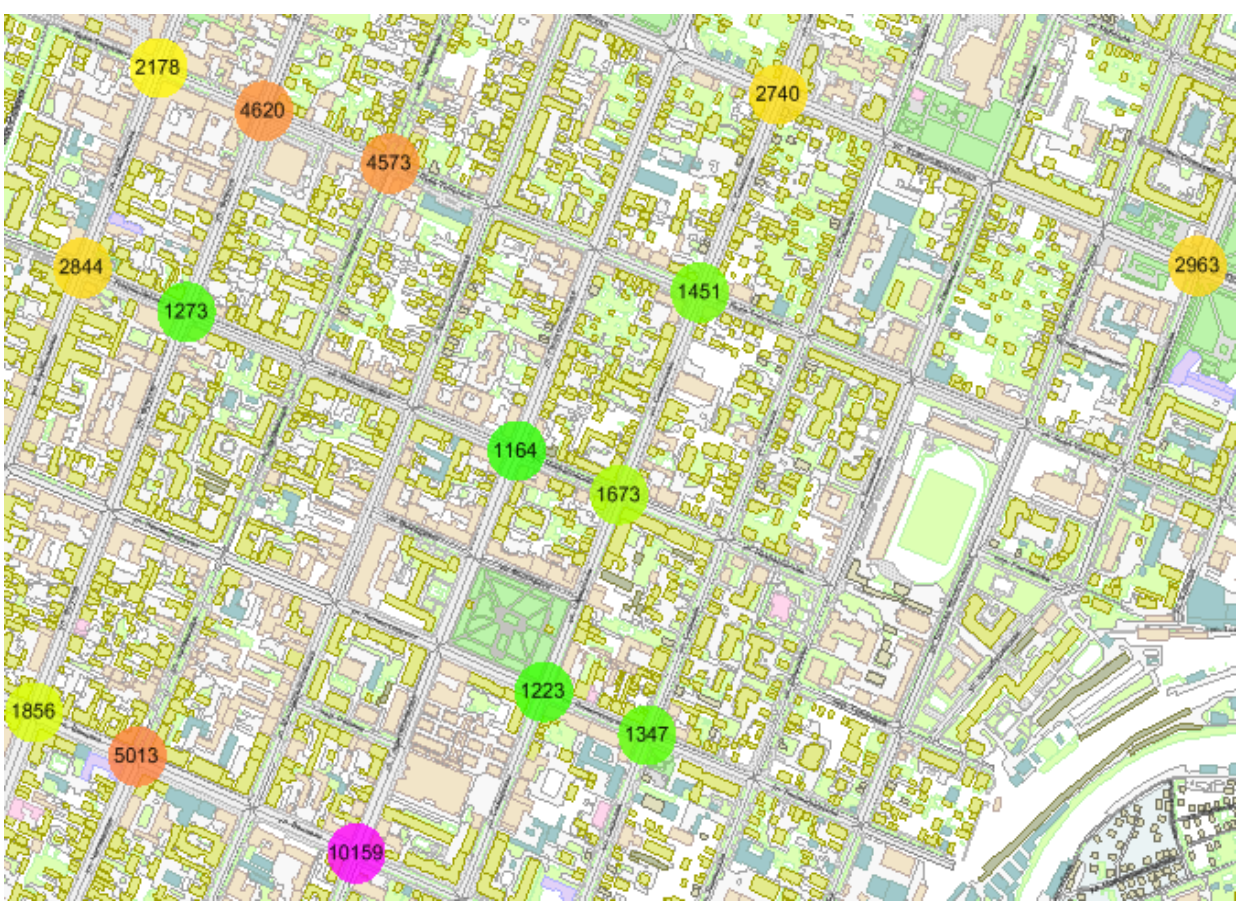

Fig. 7. Annual average daily intensity of transport flows. 


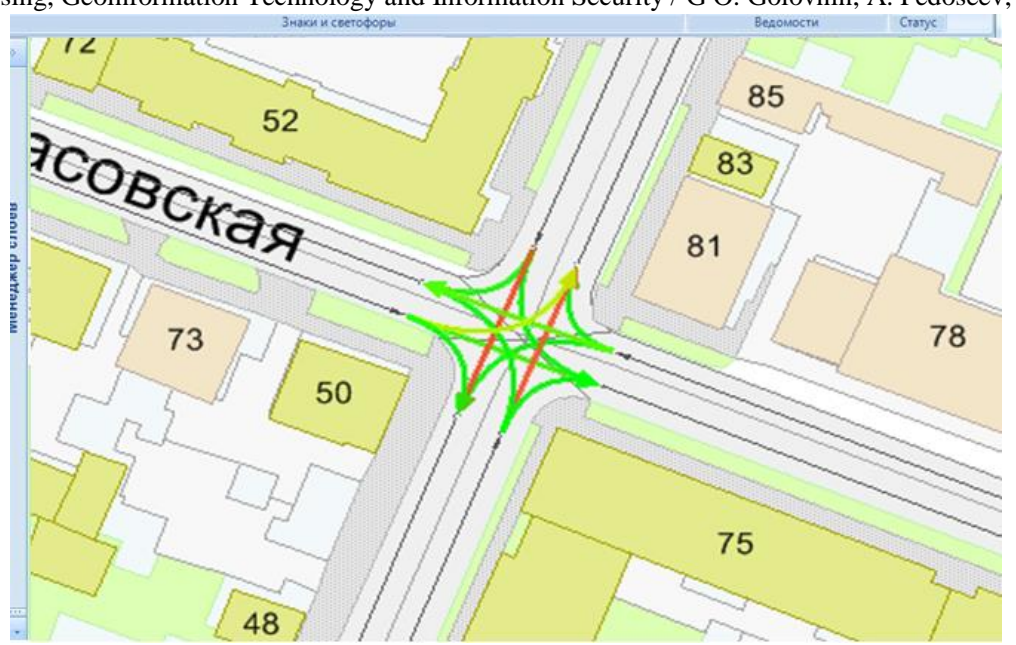

Fig. 8. Intensity of transport flows of a local crossroad.

\section{Results and Discussion}

The expected results from the implementation of the platform for transport process analysis for the cities of Surgut, Trekhgorny, Oktyabrsk and the rural settlement of Kinel-Cherkasy (Russia) are shown in Fig. 9 and Fig. 10.

The number of accident clusters (Fig. 9) is calculated using the developed PluginDTP and PluginFirecenter subsystems.

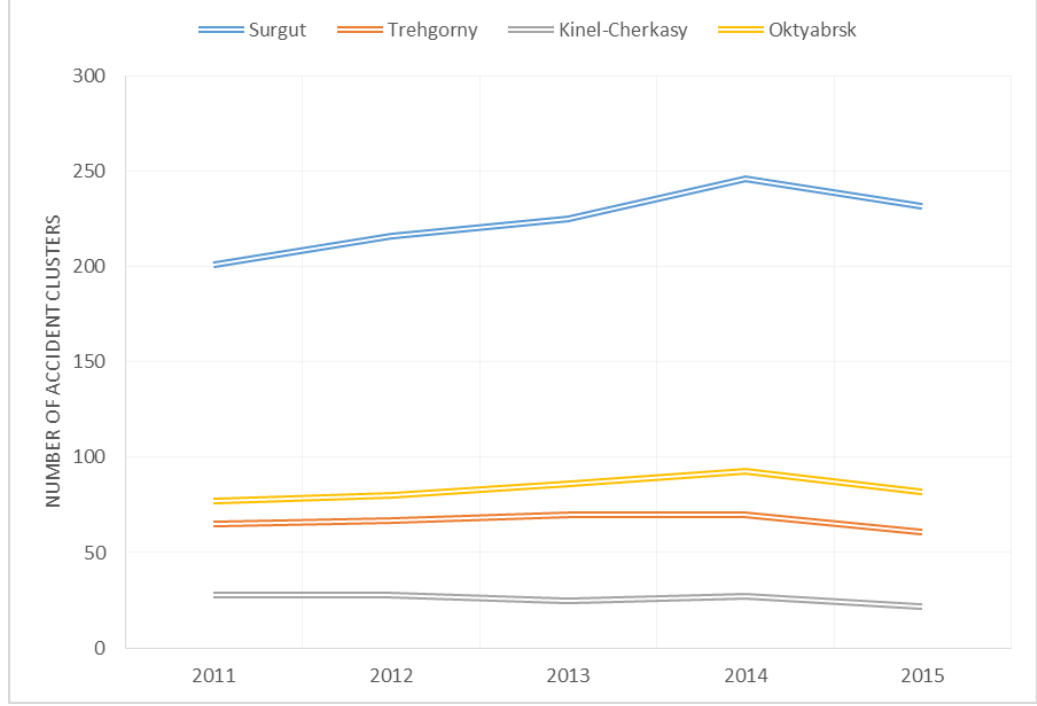

Fig. 9. Number of accident clusters.

The effect of reducing the transport delay will be obtained by reducing the time of movement of vehicles along sections of the street-road network. The data were obtained as a result of modeling the transport situation in the developed mesomodeling subsystem in the ITSGIS environment (Fig. 10).

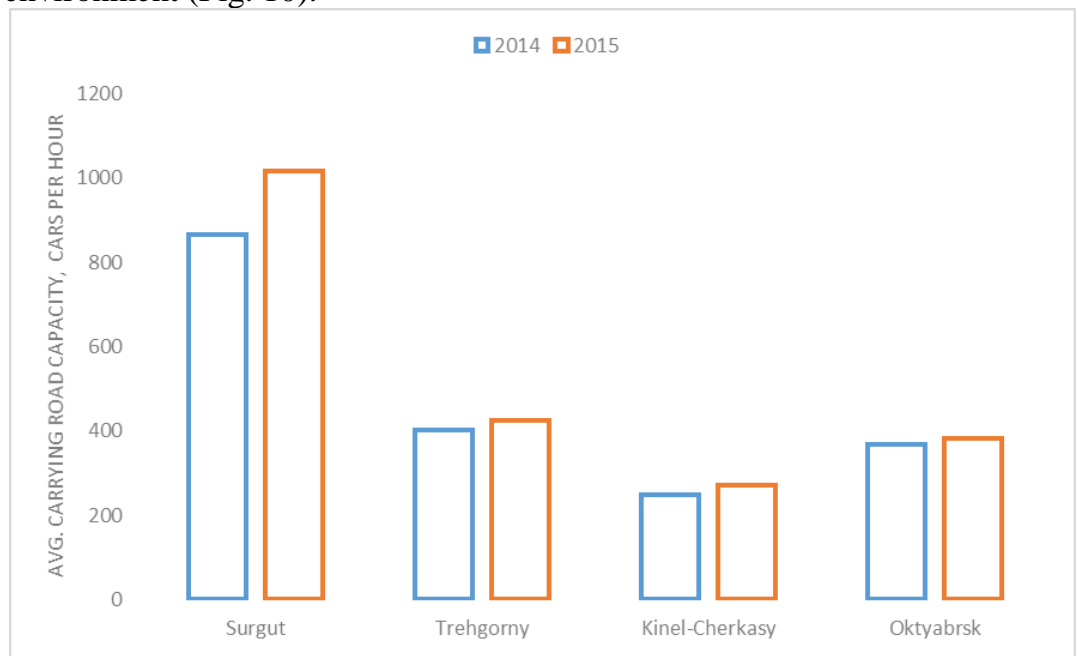

Fig. 10. Average carrying road capacity. 
Image Processing, Geoinformation Technology and Information Security / G O. Golovnin, A. Fedoseev, T. Mikheeva

The main result of the research is the introduction of the developed algorithms, tools and software into the practice of transport process management on an urbanized territory. Theoretical and practical results of the work related to the creation of the intelligent geoinformation platform for transport process analysis were applied in the following works at the science and production center "Intelligent Transport Systems" in 2011-2016: "Establishment of the municipal geoinformation system of the Samara City Administration in the part of creating an applied GIS of the Department of Improvement and Ecology", "Development of the road traffic management project in Oktyabrsk", "Preparation of initial data for creating an electronic transport model of Samara", "Preparation of initial data for calibration of the transport model of Samara", "Adjustment of the road traffic management project on the roads of Surgut", "Development of road traffic management project in Trekhgorny (Chelyabinsk region)", "Development of an integrated scheme for traffic management in the rural settlement of KinelCherkasy".

The validity of the work results is confirmed by the correct use of theoretical and experimental methods, basing on the fundamental works of domestic and foreign scientists, approbation of research results in practice, deployment in the science and production center "Intelligent Transport Systems" and in the Samara University.

\section{Conclusion}

We developed the software and algorithms based on intelligent models and modern approaches to the distributed geoinformation systems creation in order to improve efficiency of transport process analysis. Adequacy of the developed algorithms is confirmed by the results of applying an intelligent geoinformation platform for the investigation of transport processes in Surgut (Khanty-Mansiisk autonomous district) State Traffic Safety Inspectorate of the Ministry of Internal Affairs, in Trekhgorny (Chelyabinsk region), Oktyabrsk (Samara region), Kinel-Cherkasy (Samara region) administrations.

\section{Acknowledgements}

Special thanks to science and production center "Intelligent transport systems" for providing technical base for research.

\section{References}

[1] Burkov SM, Markelov GYa, Pugachev IN. Problems of system analysis and methodology for the formation of an intelligent management system for the city's transport complex. Vestnik TSU 2013; 4(31): 83-90.

[2] Izyumsky AA, Kotenkova IN. Problems and perspective directions of development of intelligent transport systems in Russia. Modernization and scientific research in the transport sector 2013; 2: 206-211.

[3] Kolosz B, Grant-Muller S, Djemame K. Modelling uncertainty in the sustainability of Intelligent Transport Systems for highways using probabilistic data fusion. Environmental Modelling \& Software 2013; 49: 78-97.

[4] Gatiyatullin Kh, Zagidullin RR. Intelligent transport system for large cities. Vestnik NCBZhD 2010; 5: 76-82.

[5] Chong Y, Quek C, Loh P. A novel neuro-cognitive approach to modeling traffic control and flow based on fuzzy neural techniques. Expert Systems with Applications 2009; 36(3): 4788-4803.

[6] Posmitny EV, Medovshchikov MI. The method of adaptive control of high-intensity traffic flows in city conditions on the basis of the meso model of dynamics using genetic algorithms. Scientific journal of KubSAU 2012; 84(10): 1-11.

[7] Robinson VB. On fuzzy sets and the management of uncertainty in an intelligent geographic information system. Recent Issues on Fuzzy Databases. Physica-Verlag HD, 2000; 109-127.

[8] Skvortsov AV, Boykov VN. Common data environment as a key element of the information modeling of highways. CAD and GIS of highways 2015; 2(5): $37-41$.

[9] Mikheeva TI. Construction of mathematical models of the city road network with the use of geoinformation technologies. Information Technologies 2006; 1 : 69-75.

[10] Skvortsov AV, Pospelov PI, Kotov AA. Geoinformatics in the road sector. Moscow: MADI, 2005; 250 p.

[11] Christodoulo OI. A joint description of spatial and attributive data based on multidimensional information objects. Software products and systems 2011; 3(95): 48-54.

[12] Kotsab M, Raday K. Integration of cartographic data into a single information system. Geodesy, cartography and aerial photography 2013; 78:127-131.

[13] Kotikov YuG. ArcGIS in models of transport systems of megacities. ArcReview, Data+, ESRI 2013; 64: 18-19.

[14] Pavlov CV, Efremova OA, Sokolova AV. The formalized description of spatial information in the composition of three-dimensional models of potentially dangerous objects on the basis of the set-theoretic approach. Electrical and information systems and systems 2014: 10(1): 66-72.

[15] Mikheeva TI, Klyuchnikov VA, Golovnin OK. Methods and procedures of transport infrastructure survey. Modern problems of science and education 2014; 6. URL: http://www.science-education.ru/120-16656.

[16] Mikheeva TI, Mikheev SV, Golovnin OK. Method of synthesis of zonal network-centric transport management system. Proceedings of the Samara Scientific Center of the RAS 2016; 4(4): 799-807.

[17] Golovnin OK, Sidorov AV, Mikhailov DA. Support of decision-making of the automatic dislocation of transport infrastructure geoobjects. Proceedings of the Samara Scientific Center of the RAS 2014; 4(2): 413-418.

[18] Fedoseev AA, Mikheev SV, Golovnin OK. Data mining in problems of trasnport infrastructure development forecasting. Modern problems of science and education 2013; 1. URL: http://www.science -education.ru/107-8153.

[19] Vasiliev SN, Oparin GA, Feoktistov AG, Sidorov IA. Intelligent technologies and tools for creating computing infrastructure in the Internet. Computational technologies 2006; S8(11): 34-44.

[20] Belyakov SL, Bozhenyuk AV, Rosenberg IN. Adaptation of the procedure for visualization of spatial data by geoinformation services. Izvestia of SFU 2015; 3(164): 248-265.

[21] Mikheev SV, Sidorov AV, Golovnin OK, Mikhailov DA. Architecture of geoinformation reference system of urban infrastructure objects. Modern problems of science and education 2013; 3.URL: http://www.science-education.ru/109-9608.

[22] Open Geospatial Consortium Web Site. URL: http://www.opengeospatial.org (16.01.2017).

[23] Geographic information system ITSGIS Web Site. URL: http://itsgis.ru/ (16.01.2017).

[24] Open Street Map Web Site. URL: http://www.openstreetmap.org (16.01.2017). 